The INL is a

U.S. Department of Energy

National Laboratory

operated by

Battelle Energy Alliance

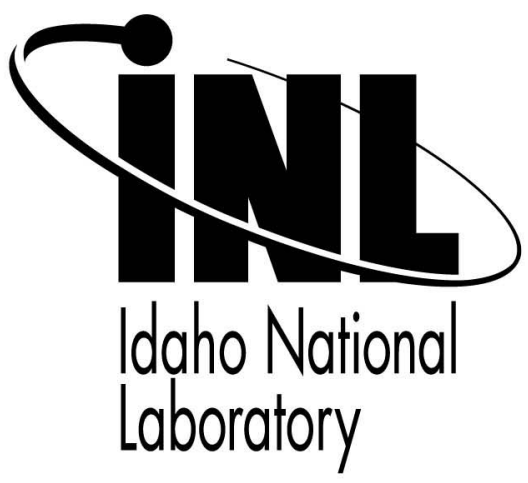

\section{CYNOD: A Neutronics Code for Pebble Bed Modular Reactor Coupled Transient Analysis}

\author{
HTR 2008
}

Hikaru Hiruta

Abderrafi M. Ougouag

Hans D. Gougar

Javier Ortensi

David W. Nigg

Cliff B. Davis

Walter L. Weaver III

\section{September 2008}

This is a preprint of a paper intended for publication in a journal or proceedings. Since changes may be made before publication, this preprint should not be cited or reproduced without permission of the author. This document was prepared as an account of work sponsored by an agency of the United States Government. Neither the United States Government nor any agency thereof, or any of their employees, makes any warranty, expressed or implied, or assumes any legal liability or responsibility for any third party's use, or the results of such use, of any information, apparatus, product or process disclosed in this report, or represents that its use by such third party would not infringe privately owned rights. The views expressed in this paper are not necessarily those of the United States Government or the sponsoring agency. 


\section{CYNOD: A NEUTRONICS CODE FOR PEBBLE BED MODULAR REACTOR COUPLED TRANSIENT ANALYSIS}

\author{
Hikaru Hiruta \\ Idaho National Laboratory \\ Idaho Falls, Idaho, USA
}

\author{
Abderrafi M. Ougouag \\ Idaho National Laboratory \\ Idaho Falls, Idaho, USA
}

\author{
Hans D. Gougar \\ Idaho National Laboratory \\ Idaho Falls, Idaho, USA
}

\author{
Cliff B. Davis \\ Idaho National Laboratory \\ Idaho Falls, Idaho, USA
}

\author{
Javier Ortensi \\ Idaho National Laboratory \\ Idaho Falls, Idaho, USA
}

\author{
David W. Nigg \\ Idaho National Laboratory \\ Idaho Falls, Idaho, USA
}

\author{
Walter L. Weaver III \\ Idaho National Laboratory \\ Idaho Falls, Idaho, USA
}

\begin{abstract}
In this paper, a new neutron kinetics solver for cylindrical $\mathrm{R}-\mathrm{Z}$ geometry, CYNOD, is presented for the simulation of coupled transient problems for pebble bed reactors. The code utilizes the Direct Coarse Mesh Finite Difference method, in which a set of one-dimensional equations in each transverse direction is solved by means of the analytic Green's function method. A method that deals with control rod cusping problems is also presented. A heterogeneous fuel kernel model is implemented in order to accurately take into account Doppler feedback effects. Numerical results that demonstrate the accuracy of the code are also presented.
\end{abstract}

\section{INTRODUCTION}

The Pebble Bed Reactor (PBR) is one of two concepts currently considered for development into the Next Generation Nuclear Plant (NGNP). Interest in the PBRs is due, in particular, to the concept's inherent safety characteristics. In order to verify and confirm the design safety characteristics of the PBR, computational tools must be developed that treat the range of phenomena that are expected to be important for this type of reactor.

A new reactor kinetics code, CYNOD, has been written based on a fixed source solver extracted from the nodal cylindrical geometry solver contained within the PEBBED code [1]. The new code thus inherits the spatial solver characteristics of the nodal solver within PEBBED. The timedependent neutron diffusion equation expressed analytically in each node of the R-Z cylindrical geometry sub-domain (or node) is transformed into one-dimensional equations by means of the usual transverse integration procedure. The onedimensional diffusion equations in each of the directions are then solved using the analytic Green's function method $[2,3]$. The resulting equations for the entire domain are then re-cast in the form of the Direct Coarse Mesh Finite Difference (DCMFD) [9] for convenience of solution.

In order to resolve the control-rod cusping effect caused by nodes that contain a partially inserted control rod, a method that takes advantage of the Green's function solution is implemented. In this corrected treatment, the nodes are homogenized using axial flux shapes reconstructed based on the Green's function method.

In steady state cases and in most slow transient problems, the homogeneous treatment of fuel zones $(5 \mathrm{~cm}$ in diameter) associated with each pebble is sufficient since the fuel temperature is very close to the temperature of the surrounding graphite due to the high thermal conductivity and the small difference in material properties between graphite and kernel coating. However, in the case of fast transient problems, involving large power excursions, the difference between fuel and graphite temperatures is significant. Thus a heterogeneous fuel kernel model is necessary for accurately computing temperatures and capturing Doppler feedback effect [7]. 
This paper presents a recently developed 2D R-Z cylindrical nodal kinetics code, CYNOD, and shows some of its capabilities by applying it to a set of known and relevant benchmarks. The new code has been coupled to the thermal hydraulics codes THERMIX/KONVEK [4] and RELAP5-3D ${ }^{\odot}$ [5] for application to the simulation of very fast transients in PBRs.

\section{NOMENCLATURE}

Areas, Volumes, and Time-Steps

$V_{r, l} \equiv$ Radial area of $l^{t h}$ radial node

$V_{z, k} \equiv$ Axial size of $k^{\text {th }}$ axial node

$\Delta t_{n} \equiv$ The $n^{\text {th }}$ time-step $\left(=t_{n+1}-t_{n}\right)$

Group Cross Sections and Delayed Neutron Parameters $\Sigma_{r}^{g} \equiv$ Removal cross section as a function of space

$\Sigma_{s 0}^{g^{\prime} \rightarrow g} \equiv$ Scattering cross section as a function of space

$\Sigma_{f}^{g} \equiv$ Fission cross section as a function of space

$\sum_{r, l, k}^{g} \equiv$ Homogenized removal cross section

$\sum_{s, l, l, k}^{g^{\prime} \rightarrow g} \equiv$ Homogenized scattering cross section

$\Sigma_{f, l, k}^{g} \equiv$ Homogenized fission cross section

$v^{g} \equiv$ Number of fission neutron per fission

$\mathrm{v}^{g} \equiv$ Neutron speed

$\beta_{i} \equiv$ Delayed neutron fraction for the $i^{\text {th }}$ precursor

$\bar{\beta} \equiv \sum_{i=1}^{I_{D}} \beta_{i}$

$\lambda_{i} \equiv$ Decay constant for the $i^{\text {th }}$ delayed neutron precursor

$\chi_{p}^{g} \equiv$ Prompt fission neutron spectra

$\chi_{D, i}^{g} \equiv$ Delayed neutron spectra

\section{Fluxes and sources}

$\phi^{g} \equiv$ Neutron scalar flux

$J_{r}^{g} \equiv$ Radial net current

$J_{z}^{g} \equiv$ Axial net current

$C_{i} \equiv$ Delayed neutron precursor concentration

$\Phi_{l, k}^{g} \equiv$ Node averaged scalar flux

$J_{r, l, k}^{g, s} \equiv$ Radial interface current on surface $s$ of node $(l, k)$

$J_{z, l, k}^{g, s} \equiv$ Axial interface current on surface $s$ of node $(l, k)$

$C_{i, l, k} \equiv$ Node averaged precursor concentration

$S_{r, l, k}^{g} \equiv$ Node average source term for radial direction

$S_{z, l, k}^{g} \equiv$ Node average source term for axial direction

$S_{l, k, m}^{g} \equiv$ The $m^{\text {th }}$ axial spatial source moment

\section{Functions and Coefficients}

$G_{l, k}^{g}\left(z, z^{\prime}\right) \equiv$ Green's function for axial direction

$G_{r, \pm l, k}^{g} \equiv$ Green's function for radial direction

$\left(G_{ \pm, l, k}^{g}\right)_{m} \equiv$ The $m^{\text {th }}$ axial spatial moment of Green's

Function

$D_{l, k}^{g, T / B}, C_{l, k}^{f, g} \equiv$ Axial spatial coupling coefficients

$D_{l, k}^{g, R / L}, \Lambda_{l, l+1, k}^{g} \equiv$ Radial spatial coupling coefficients

\section{METHODOLOGY}

Direct CMFD (D-CMFD) Method for Time-Dependent Diffusion Equation in Cylindrical Geometry

In this section, we present the D-CMFD method for solution of the time-dependent diffusion equation in cylindrical (R-Z) geometry based on the Green's function method, which was originally developed for the PEBBED steady-state solver.

Let us consider the time-dependent form of the few-group neutron balance equation in cylindrical geometry:

$$
\begin{aligned}
& \frac{1}{\mathrm{v}^{g}} \frac{\partial \phi^{g}}{\partial t}+\frac{1}{r} \frac{\partial}{\partial r} r J_{r}^{g}+\frac{\partial}{\partial z} J_{z}^{g}+\sum_{r}^{g} \phi^{g}= \\
& \sum_{g^{\prime} \neq g}^{G} \sum_{s 0}^{g^{\prime} \rightarrow g} \phi^{g^{\prime}}+(1-\bar{\beta}) \chi_{p}^{g} \sum_{g^{\prime}=1}^{G} v^{g^{\prime}} \sum_{f}^{g^{\prime}} \phi^{g^{\prime}}+\sum_{i=1}^{I_{D}} \chi_{D, i}^{g} \lambda_{i} C_{i}
\end{aligned}
$$

and a set of equations for delayed neutron precursors given by:

$\frac{\partial C_{i}}{\partial t}=\beta_{i} \sum_{g=1}^{G} v^{g} \sum_{f}^{g} \phi^{g}-\lambda_{i} C_{i}$ for $i=1, \ldots, I_{D}$,

where all notations are given in the previous section. In the case of eigenvalue initiated transient calculations, all fission source terms in Eqs. (1) and (2) are scaled by $k_{\text {eff. }}$ To derive a set of discretized balance equations, we integrate Eq. (1) over the volume of node $(l, k)\left(a_{l} \leq r \leq b_{l},-z_{k} \leq z \leq z_{k}, l=1, \ldots, N_{l}, k=\right.$ $\left.1, \ldots, N_{k}\right)$ :

$$
\begin{gathered}
\int_{a_{l}}^{b_{l}} \int_{z_{k}}^{z_{k}}\left(\frac{1}{v^{g}} \frac{\partial \phi^{g}}{\partial t}+\frac{1}{r} \frac{\partial}{\partial r} r J_{r}^{g}+\frac{\partial}{\partial z} J_{z}^{g}+\sum_{r}^{g} \phi^{g}-\sum_{g^{\prime} \neq g}^{G} \Sigma_{s 0}^{g^{\prime} \rightarrow g} \phi^{g^{\prime}}\right. \\
\left.-(1-\bar{\beta}) \chi_{p}^{g} \sum_{g^{\prime}=1}^{G} v^{g^{\prime}} \sum_{f}^{g^{\prime}} \phi^{g^{\prime}}-\sum_{i=1}^{I_{D}} \chi_{D, i}^{g} \lambda_{i} C_{i}\right) r d r d z=0
\end{gathered}
$$

Then, assuming that the cross sections are piece-wise constant on the set of nodes (i.e., homogenized), we obtain a set of discretized nodal balance equations in R-Z geometry:

$$
\begin{gathered}
\frac{1}{\mathrm{v}^{g}} \frac{\partial \Phi_{l, k}^{g}}{\partial t}+\frac{b_{l}}{V_{r, l}} J_{r, l, k}^{g, R}-\frac{a_{l}}{V_{r, l}} J_{r, l, k}^{g, L}+\frac{1}{V_{z, k}} J_{z, l, k}^{g, T}- \\
\frac{1}{V_{z, k}} J_{z, l, k}^{g, B}+\sum_{r, l, k}^{g} \Phi_{l, k}^{g}=\sum_{g^{\prime} \neq g}^{G} \sum_{\substack{g_{0, l, k}^{\prime} \\
g^{\prime}}}^{g} \Phi_{l, k}^{g^{\prime}}+ \\
(1-\bar{\beta}) \chi_{p}^{g} \sum_{g^{\prime}=1}^{G} v^{g^{\prime}} \sum_{f, l, k}^{g^{\prime}} \Phi_{l, k}^{g^{\prime}}+\sum_{i=1}^{I_{D}} \chi_{D, i}^{g} \lambda_{i} C_{i, l, k}
\end{gathered}
$$

In order to derive a set of equations as a function of the node average scalar flux $\Phi_{l, k}^{g}$, we derive expressions for interface 
currents $J$ as a function of $\Phi_{l, k}^{g}$ in adjacent nodes by means of Analytic Nodal Greens' Function Method (ANGFM) [2,3]:

$$
\begin{aligned}
& J_{r, l, k}^{g, R}=D_{l+1, k}^{g, R} \Phi_{l+1, k}^{g}-D_{l, k}^{g, L} \Phi_{l, k}^{g}-\frac{D_{l+1, k}^{g, R}}{\sum_{r, l+1, k}^{g}} S_{r, l+1, k}^{g} \\
& -\frac{D_{l, k}^{g, L}}{\sum_{r, l, k}^{g}} S_{r, l, k}^{g}-\frac{1}{\Lambda_{l, l+1, k}^{g}} G_{r,-, l+1, k}^{g} S_{r, l+1, k}^{g}+\frac{1}{\Lambda_{l, l+1, k}^{g}} G_{r,+, l, k}^{g} S_{r, l, k}^{g}
\end{aligned}
$$

for the $r$-direction, and

$$
\begin{aligned}
J_{z, l, k}^{g, T} & =-D_{l, k+1}^{g, T} \Phi_{l, k+1}^{g}+D_{l, k}^{g, B} \Phi_{l, k}^{g}+\frac{D_{l, k+1}^{g, T}}{\sum_{r, l, k+1}^{g}} S_{z, l, k+1}^{g}-\frac{D_{l, k}^{g, B}}{\sum_{r, l, k}^{g}} S_{z, l, k}^{g} \\
& -\frac{D_{l, k+1}^{g, T}}{C_{l, k+1}^{f, g}} \sum_{m=1}^{M}\left(G_{-, l, k+1}^{g}\right)_{m} S_{l, k+1, m}^{g}+\frac{D_{l, k}^{g, B}}{C_{l, k}^{g, g}} \sum_{m=1}^{M}\left(G_{+, l, k}^{g}\right)_{m} S_{l, k, m}^{g}
\end{aligned}
$$

for the $z$-direction. Note that the expressions for interface currents on the other surfaces are similar to Eqs. (5) and (6).

The numerical treatment of the time dependence in neutron kinetics is a difficult task since the equations are stiff due to the great difference in the time constants associated with prompt neutron production and delayed neutron precursor decay. In order to deal with such difficulties, one needs a robust and stable method. The time derivative is discretized by means of the implicit Euler method:

$$
\left.\frac{\partial \Phi_{l, k}^{g}}{\partial t}\right|_{t=t_{n+1}} \cong \frac{\Phi_{l, k}^{g}\left(t_{n+1}\right)-\Phi_{l, k}^{g}\left(t_{n}\right)}{\Delta t_{n}}
$$

which ensures unconditional numerical stability.

For the discretization of the delayed neutron precursor equations given by Eq. (2), we utilize the integrating factor method:

$$
C_{i, l, k}\left(t_{n+1}\right)=C_{i, l, k}\left(t_{n}\right) e^{-\lambda_{i} \Delta t_{n}}+\beta_{i} \int_{t_{n}}^{t_{n+1}} \sum_{g=1} v^{g} \sum_{f, l, k}^{g} \Phi_{l, k}^{g}\left(t^{\prime}\right) e^{-\lambda_{i}\left(t_{n+1}-t^{\prime}\right)} d t^{\prime}
$$

If we assume that the fission source varies linearly between time steps, then we can evaluate the integral term in Eq. (8) as:

$$
\begin{array}{r}
C_{i, l, k}\left(t_{n+1}\right)=C_{i, l, k}\left(t_{n}\right) e^{-\lambda_{i} \Delta t_{n}}+H_{i_{n}, l, k}^{0, n} \sum_{g=1} v^{g} \sum_{f, l, k}^{g} \Phi_{l, k}^{g}\left(t_{n}\right)+ \\
H_{i_{n}, l, k}^{1, n} \sum_{g=1} v^{g} \sum_{f, l, k}^{g} \Phi_{l, k}^{g}\left(t_{n+1}\right)
\end{array}
$$

where

$$
\begin{aligned}
& H_{i, l, k}^{1, n}=\frac{\beta_{i}}{\lambda_{i} \Delta t_{n}}\left(\Delta t_{n}-\frac{1}{\lambda_{n}}\left(1-e^{-\lambda_{i} \Delta t_{n}}\right)\right), \\
& H_{i, l, k}^{0, n}=-H_{i, l, k}^{1, n}+\frac{\beta_{i}}{\lambda_{i}}\left(1-e^{-\lambda_{i} \Delta t_{n}}\right)
\end{aligned}
$$

If we substitute Eqs.(5)-(7) and (9) into Eq.(4), we obtain a the system of equations whose coefficients can be written in the form of a five-banded matrix. At each time-step, this system of equations is numerically solved by inner and outer iteration loops (see Figure 1). In the inner iteration loop, the transverse leakage terms are updated, and the system of equations with the five-banded coefficient matrix is inverted by means of the Line-SOR scheme. The outer iteration updates the fission terms based on the solution obtained at the previous iteration. During the outer iteration, the solution is extrapolated by means of an asymptotic source extrapolation method [6]:
$\Phi^{(j+1)}=\Phi^{(j+1 / 2)}+\frac{\sigma}{1-\sigma}\left(\Phi^{(j+1 / 2)}-\Phi^{(j)}\right)$

where $j$ is the outer iteration index. (Note: The spatial and temporal indices are suppressed). This acceleration scheme requires calculation of $\sigma$, which can be reasonably estimated by taking the ratio of error norms of two successive iterations:

$$
\sigma \cong \frac{\left\|\Phi^{(l+1)}-\Phi^{(l)}\right\|}{\left\|\Phi^{(l)}-\Phi^{(l-1)}\right\|}
$$

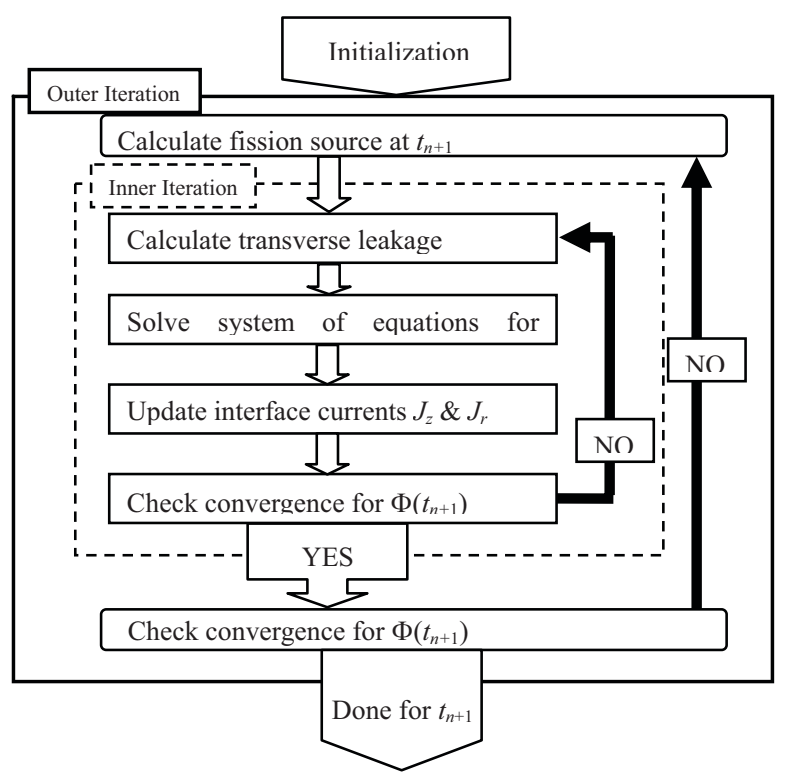

Figure 1: Flowchart for the iterative process in CYNOD.

\section{Treatment of Control-Rod Cusping Problem}

This section presents the method implemented for resolving the control rod cusping problem. The rod-cusping problem arises when a control rod is partially inserted into a large spatial node, and the calculation exhibits unphysical cusps in reactivity plots. One way to resolve this problem is to obtain homogenized cross sections by correctly mixing rodded and un-rodded zones. To perform such homogenization, we reconstruct the axial shape of the group scalar flux within a partially rodded node at each time-step by means of ANGFM:

$\Phi_{L, K}^{g}\left(z, t_{n}\right)=G_{L, K}^{g}\left(z,-z_{K}\right) J_{z, L, K}^{g, B}-G_{L, K}^{g}\left(z, z_{K}\right) J_{z, L, K}^{g, T}+\sum_{m=1}^{M}\left(G_{L, K}^{g}(z)\right)_{m} S_{L, K, m}^{g}$,

where the indices $L$ and $K$ correspond to the radial and axial positions of the node containing a partially inserted control rod, respectively. Using Eq. (10), we calculate both control-rod zone and graphite zone averaged scalar fluxes by integrating it over each zone:

$$
\Phi_{L, K}^{g, C R}\left(t_{n}\right)=\int_{z_{C R}}^{z_{K}} \Phi_{L, K}^{g}\left(z, t_{n}\right) d z
$$




$$
\Phi_{L, K}^{g, \text { graphite }}\left(t_{n}\right)=\int_{-z_{K}}^{z_{C R}} \Phi_{L, K}^{g}\left(z, t_{n}\right) d z,
$$

where $z_{C R}$ is the bottom surface of the control-rod. Then, the homogenized cross sections are calculated by utilizing zone averaged scalar fluxes as weights:

$$
\Sigma_{\text {hom }}^{g}\left(t_{n}\right)=\frac{\Sigma_{C R}^{g}\left(t_{n}\right) \Phi_{L, k}^{g, C R}\left(t_{n}\right) \Delta z_{k}^{C R}+\sum_{\text {graphite }}^{g}\left(t_{n}\right) \Phi_{L, k}^{g, \text { graphite }}\left(t_{n}\right) \Delta z_{k}^{\text {graphite }}}{\Phi_{L, k}^{g, C R}\left(t_{n}\right) \Delta z_{k}^{C R}+\Phi_{L, k}^{g, g r a p h i t e}\left(t_{n}\right) \Delta z_{k}^{\text {graphite }}}
$$

where

$\Delta z_{k}^{C R}=z_{k}-z_{C R}, \Delta z_{k}^{\text {graphite }}=z_{C R}+z_{k}$

\section{Coupling with Thermal-Hydraulic Codes}

The coupled codes CYNOD-RELAP5-3D ${ }^{\odot}$ and CYNODTHERMIX/KONVEK have been developed for the simulation of PBR transients. The flowchart of the coupled transient simulation process is shown in Figure 2. The neutronics and thermal hydraulic codes are connected by an interface routine that performs communication of data (power and temperature distributions, time steps, etc.) from each code. In particular, for coupling with RELAP5-3D, the interface routine is replaced with the general purpose code PVMEXEC [10], which serves as a message passing interface between CYNOD and RELAP53D. The PVMEXEC program operates under the PVM environment, performing (1) startup of each simulation code, (2) synchronization of coupled simulation process, (3) classification of messages sent/received by each code, and (4) termination of coupled simulations. By using this program, one can keep CYNOD and RELAP5-3D as separate programs instead of creating one massive coupled code.

The coupled simulation starts from the neutronics calculation by CYNOD. The calculated power density distribution from CYNOD is transferred to the thermal hydraulic code for the calculation of bulk graphite temperature and homogenized fuel temperature at the advanced time-step. Utilizing the bulk graphite temperature and the power density distribution renormalized to the fuel kernel volume, a heterogeneous temperature module calculates average fuel kernel (Doppler) temperature. Within the heterogeneous temperature module, an iterative scheme for the fuel kernel temperature is implemented. The iteration consists of calculations of temperature dependent conductivity/heat transfer coefficient in each layer of the fuel particle and calculations/convergence check of the average fuel kernel temperature. Based on the obtained fuel kernel and bulk graphite temperatures, cross sections are linearly interpolated from the library and passed to CYNOD for the next time-step. The time-steps of CYNOD-RELAP5-3D are determined by means of the material Courant time step stability limit. The same time steps are also used for the CYNODTHERMIX/KONVEK simulation for consistent comparisons.

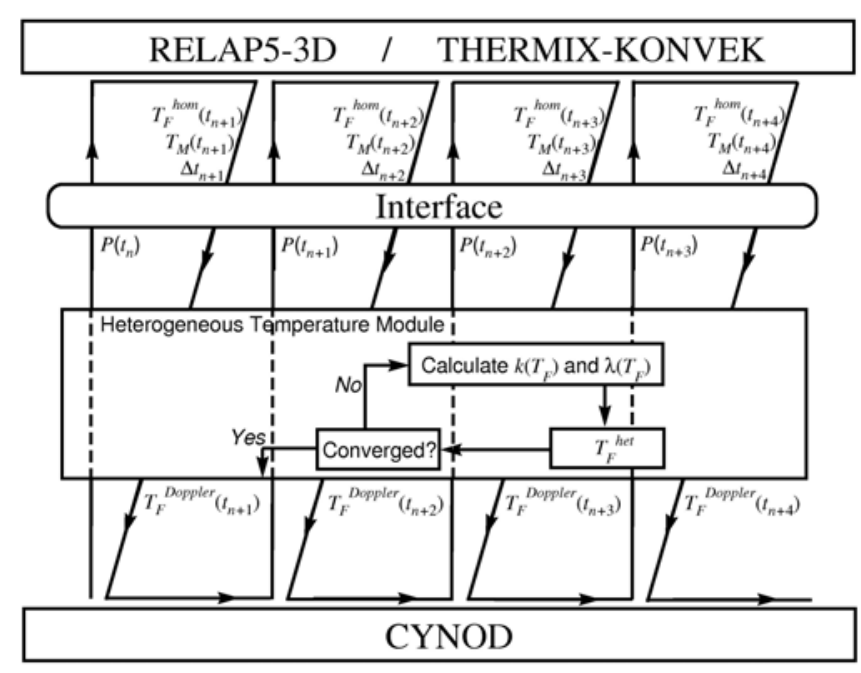

Figure 2: Flowchart of coupled CYNOD-THERMIX full-core calculations

\section{RESULTS AND DISCUSSIONS}

The performance of the standalone CYNOD transient code was evaluated using Dodds's R-Z benchmark problem [8]. This test problem is a 2-D $(r-z)$, delayed supercritical transient diffusion theory problem with two neutron energy groups and six delayed neutron groups. The problem geometry is shown in Figure 3. In this problem, the thermal absorption cross section in regions 3 and 7 is linearly increased by $3 \%$ and is linearly decreased by $3 \%$ in region 11 , over a period of 1 second in both cases. The relative power level calculated by the code is shown in Figure 4, along with the reference values. The figure shows multiple cases corresponding to various discretization refinement levels of the time and spatial variables. The results agree very well when the quadratic source approximation in the nodal equations is used in the axial direction. However, when a flat source is used, the solution does not achieve the same quality of accuracy as that obtained by using quadratic source, even if the number of axial mesh intervals is doubled.

To further demonstrate the capability of the coupled code, we present the fast rod ejection problem from the OECD PBMR-400 benchmark. In this test problem, all control rods within the outer reflector are pulled out $200 \mathrm{~cm}$ above their original position over 0.1 second. Figures 5 and 6 show comparisons of relative power levels, and fuel and moderator temperatures obtained by CYNOD-REALP5-3D and CYNODTHERMIX/KONVEK, respectively. According to these two figures, results obtained by these two coupled codes are consistent. The rapid increase of fuel temperature in the developed heterogeneous fuel kernel model prevents further increase of power level, accurately accounting for the Doppler effect. Slightly higher fuel and moderator temperatures are observed in CYNOD-RELAP5-3D results. The less detailed axial nodalization in RELAP5-3D causes the average temperatures to be somewhat higher than in THERMIX. 


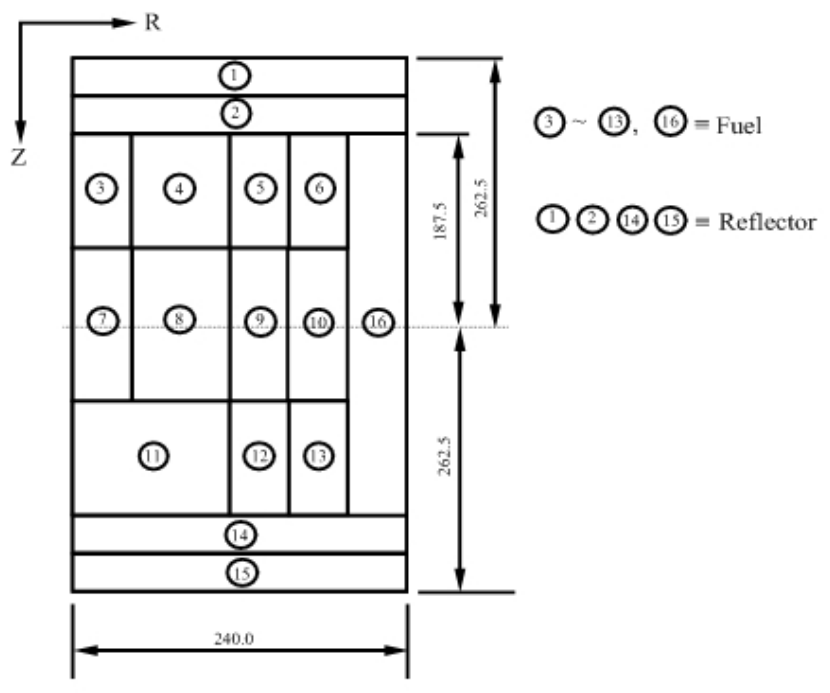

Figure 3: Schematic diagram of Dodds' delayed supercritical transient benchmark problem.

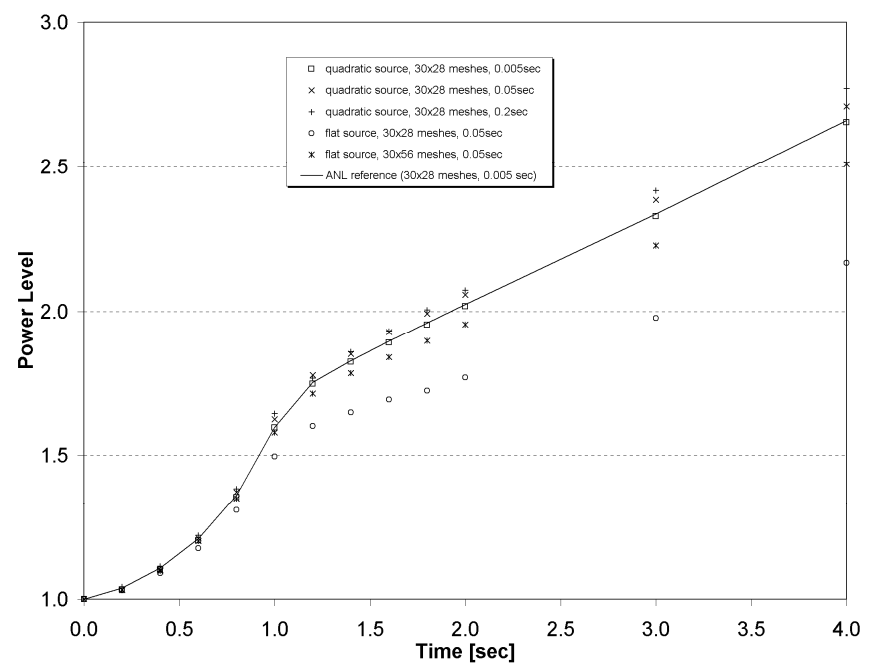

Figure 4: Comparison of relative power levels obtained by CYNOD.

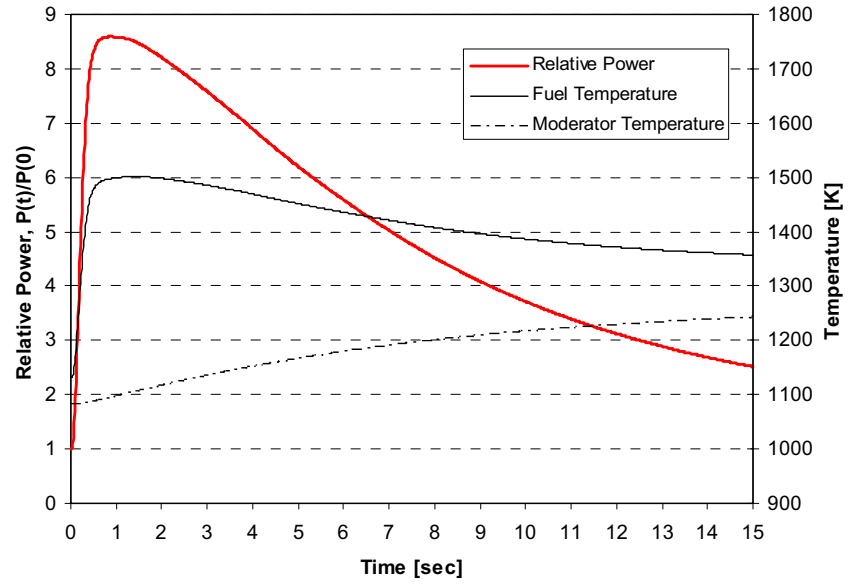

Figure 5: Comparison of relative power levels, fuel and moderator (graphite) temperatures calculated by CYNODRELAP5-3D.

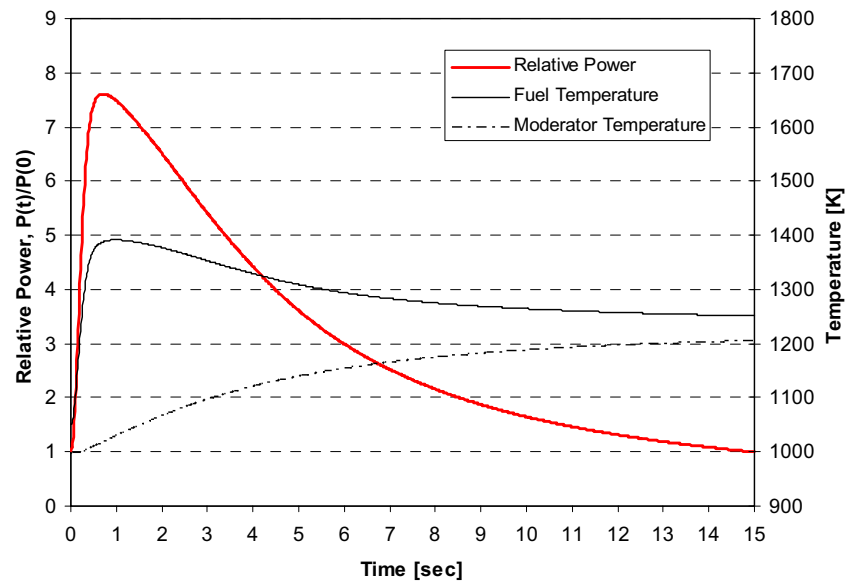

Figure 6: Comparison of relative power levels, fuel and moderator temperatures calculated by CYNODTHERMIX/KONVEK

\section{CONCLUSIONS}

A 2-D R-Z cylindrical nodal kinetics code, CYNOD, has been developed for accurate coupled transient simulations PBR cores. In this code, one-dimensional equations in each transverse direction are solved using the Analytic Nodal Green's Function Method. The resulting equations for the entire domain are then re-cast in the form of the Direct Coarse Mesh Finite Difference method. In order to resolve the control rod cusping effect, cross sections in the partially rodded nodes are homogenized using axial flux shapes reconstructed based on the Green's function method. A heterogeneous fuel kernel model is implemented in order to accurately take into account Doppler feedback effects. The numerical results for the delayed supercritical benchmark problem indicate high accuracy of the standalone kinetics code. The newly developed standalone kinetics code has been coupled with the thermal hydraulics 
codes RELAP5-3D and THERMIX/KONVEK. The results for the coupled rod-ejection transient problem show that both sets of coupled codes with the new heterogeneous temperature module accurately capture the Doppler temperature feedback effects of the coupled rapid transient problem.

\section{REFERENCES}

1. W. K. Terry, H. D. Gougar, and A. M. Ougouag, "Direct Deterministic Method for Neutronics Analysis and Computation of Asymptotic Burnup Distribution in a Recirculating Pebble-Bed Reactor," Ann. Nucl. Energy, 29, pp. 1345-1364 (2002).

2. A. M. Ougouag, "A Coarse-Mesh Nodal Method for Multigroup Multidimensional Neutron Diffusion Computations," M.S. Thesis, University of Illinois (1981).

3. H. L. Rajic, and A. M. Ougouag, "ILLICO: A Nodal Neutron Diffusion Method for Modern Computer Architectures," Nucl. Sci. Eng., 103, 392-408 (1989).

4. E. Teuchert, et al, "V.S.O.P. - Computer Code System for Reactor Physics and Fuel Cycle Simulation," Forschungszentrum Jülich GmbH Jül-2897 (1994).

5. The RELAP5-3D ${ }^{\odot}$ Code Development Team, "RELAP5-3D ${ }^{\odot}$ Code Manual Volume I: Code Structure, System Models and Solution Methods,"
INEEL-EXT-98-00834, Revision 2.4, Idaho National Laboratory (2005).

6. D. A. Meneley, "Acceleration of External Source Problems in Near-Critical Systems," Applied Physics Division Annual Report: July 1, 1970, to June 30, 1971, ANL-7910, 513-515, Argonne National Laboratory.

7. T. Rademer, W. Bernnat, and G. Lohnert, "Coupling of Neutronics and Thermal Hydraulics Codes for the Simulation of Transients of Pebble Bed HTR Reactors," $2^{\text {nd }}$ International Topical Meeting on High Temperature Reactor Technology, Beijing, China, September 22-24, (2004).

8. Argonne Code Center: Benchmark Problem Book, ANL-7416, Suppl. No. 2, Argonne National Lboratory (1977).

9. Y. A. Chao, "A Theoretical Analysis of the Coarse Mesh Finite Difference Representation in Advanced Nodal Methods," Proc. Int. Conf. Mathematics and Computation, Reactor Physics and Environmental Analysis in Nuclear Applications, Madrid, Spain (1999).

10. W. L. Weaver III, "The Application Programming Interface for the PVMEXEC Program and Associated Code Coupling System," INL/EXT-05-00107, Idaho National Laboratory (2005). 